\title{
ON FREE SPECTRA OF LOCALLY TESTABLE SEMIGROUP VARIETIES
}

\author{
IGOR DOLINKA \\ Department of Mathematics and Informatics, University of Novi Sad, \\ Trg Dositeja Obradovića 4, 21000 Novi Sad, Serbia \\ e-mail:dockie@dmi.uns.ac.rs
}

(Received 26 August 2010; accepted 30 November 2010; first published online 10 March 2011)

\begin{abstract}
For each $k \geqslant 2$, we determine the asymptotic behaviour of the sequence of cardinalities of finitely generated free objects in $\mathcal{T}_{k}$, the variety consisting of all $k$ testable semigroups.
\end{abstract}

2010 Mathematics Subject Classification. 20M07, 08B20, 05A16, 05 C20.

1. Introduction. Let $X$ be an alphabet, and let $X^{+}$denote the free semigroup on $X$, consisting of all non-void (finite) words over $X$. A semigroup $S$ satisfies the identity $\mathbf{u} \approx \mathbf{v}$, where $\mathbf{u}, \mathbf{v} \in X^{+}$, if $\phi(\mathbf{u})=\phi(\mathbf{v})$ holds in $S$ for any homomorphism $\phi: X^{+} \rightarrow S$. Let $|\mathbf{w}|$ denote the length of a word $\mathbf{w} \in X^{+}$. If $k \geqslant 2$ and $|\mathbf{u}|,|\mathbf{v}| \geqslant k$, then the identity $\mathbf{u} \approx \mathbf{v}$ is said to be $k$-testable [20, 22] if: (a) the prefixes of $\mathbf{u}$ and $\mathbf{v}$ of length $k-1$ coincide, (b) the suffixes of $\mathbf{u}$ and $\mathbf{v}$ of length $k-1$ coincide and (c) the sets of subwords of $\mathbf{u}$ and $\mathbf{v}$ of length $k$ coincide. A semigroup $S$ is $k$-testable if it satisfies all $k$-testable identities; consequently, the class of all $k$-testable semigroups is a variety, which we denote by $\mathcal{T}_{k}$. A semigroup is locally testable if it is $k$-testable for some $k$, that is, if it belongs to some of the varieties $\mathcal{T}_{k}$.

The notion of local testability originated from formal language theory, where it plays a significant role. It was first introduced by McNaughton and Papert [17], and since then it was a subject of various investigations in theoretical computer science; we refer to the survey [19] for an overview, see also [3, 14]. An algebraic treatment of this notion is also fruitful, and it is not limited exclusively to semigroup-theoretical aspects $[16,20,22]$, but pertains to the more classical setting of combinatorial group theory as well [10].

In the course of investigating a particular variety of algebras, it is often the first step to solve the word problem for free objects of that variety - this is just equivalent to the problem of algorithmic recognition of identities satisfied by the variety in question. Now assume that $\mathcal{V}$ is a locally finite variety. With effective solutions of word problems of free objects in $\mathcal{V}$ at hand, it might become possible either to explicitly determine, or, at least, to asymptotically estimate the size of $\mathfrak{F}_{n}(\mathcal{V})$, the free algebra of $\mathcal{V}$ freely generated by an $n$-element set, $n \geqslant 1$. The sequence of cardinalities $f_{n}(\mathcal{V})=\left|\mathfrak{F}_{n}(\mathcal{V})\right|$ is called the free spectrum of $\mathcal{V}$. An interesting general-algebraic fact is that the rate of growth of $f_{n}(\mathcal{V})$ is intimately connected to the structural properties of $\mathcal{V}$. For example, for a locally finite group variety $\mathcal{V}$, we have $\log _{2}\left|f_{n}(\mathcal{V})\right| \in \mathcal{O}\left(n^{c}\right)$ if and only if $\mathcal{V}$ consists of step- $c$ nilpotent groups, while otherwise $\log _{2}\left|f_{n}(\mathcal{V})\right|$ is at least exponential $[\mathbf{1 1}, \mathbf{1 8}]$. Some general-algebraic ramifications of structural features implied by free spectra may be found in $[\mathbf{2}, \mathbf{1 2}]$. Let us also mention that the free spectrum of a variety has a 
'companion', a closely related invariant called the $p_{n}$-sequence; we refer, e.g. to $[\mathbf{5}, \mathbf{6}]$ for several results and references concerning $p_{n}$-sequences of semigroups.

In this note, we determine the asymptotic class of $f_{n}\left(\mathcal{T}_{k}\right)$, the free spectrum of the variety of $k$-testable semigroups, for any $k \geqslant 2$. In this way, we put the recent note of Kátai-Urbán and Szabó [13] in a more general context, thus obtaining their result as a corollary. Namely, the note [13] is concerned with the free spectrum of the variety $\mathcal{A}$ generated by the five-element semigroup $A_{2}=\langle a, b| a^{2}=a, b^{2}=0, a b a=a, b a b=$ $b\rangle$ (see [15] for more information on the prominent role of $A_{2}$ in semigroup theory). However, by [22, Theorem 2], we have that $\mathcal{A}$ actually coincides with $\mathcal{T}_{2}$, the variety of 2-testable semigroups. Our result is now as follows.

THEOREM 1.1.

$$
f_{n}\left(\mathcal{T}_{k}\right) \sim n^{2(k-1)} 2^{n^{k}}
$$

Here, we write $a_{n} \sim b_{n}$ for two sequences $\left\{a_{n}\right\}$ and $\left\{b_{n}\right\}$ if $\lim _{n \rightarrow \infty} \frac{a_{n}}{b_{n}}=1$. Hence, [13, Theorem 4.3] is just the case $k=2$ in the above statement.

2. Preliminaries. Let $X_{n}=\left\{x_{1}, \ldots, x_{n}\right\}$. For any $r \geqslant 1$, we denote by $X_{n}^{r}$ the set of all words over $X_{n}$ of length $r$,

$$
X_{n}^{r}=\left\{x_{i_{1}} \cdots x_{i_{r}}: 1 \leqslant i_{1}, \ldots, i_{r} \leqslant n\right\} .
$$

No distinction is made between $X_{n}^{1}$ and $X_{n}$ itself. For a word $\mathbf{w} \in X_{n}^{+}$and $r \leqslant|\mathbf{w}|$, we define $h_{r}(\mathbf{w})$ to be the prefix of $\mathbf{w}$ of length $r$; analogously, $t_{r}(\mathbf{w})$ is the suffix of $\mathbf{w}$ of length $r$.

As noted in [22], there is a convenient way to express the $k$-testability of a semigroup identity $\mathbf{u} \approx \mathbf{v}$ in terms of graphs. Namely, for $\mathbf{w} \in X_{n}^{+}$let $\Gamma_{k}(\mathbf{w})$ be the directed graph whose vertices are all subwords of $\mathbf{w}$ of length $k-1$ (multiple occurrences of a subword being denoted by the same vertex), while $\mathbf{a} \rightarrow \mathbf{b}$ is an edge if and only if there is a subword $\mathbf{w}^{\prime}$ of $\mathbf{w}$ of length $k$ such that $\mathbf{a}=h_{k-1}\left(\mathbf{w}^{\prime}\right)$ and $\mathbf{b}=t_{k-1}\left(\mathbf{w}^{\prime}\right)$; equivalently, the suffix of $\mathbf{a}$ of length $k-2$ coincides with the prefix of $\mathbf{b}$ of the same length.

Lemma 2.1 ([22]). Let $\mathbf{u}, \mathbf{v} \in X_{n}^{+}$such that $|\mathbf{u}|,|\mathbf{v}| \geqslant k$. An identity $\mathbf{u} \approx \mathbf{v}$ holds in $\mathcal{T}_{k}$ if and only if $h_{k-1}(\mathbf{u})=h_{k-1}(\mathbf{v}), t_{k-1}(\mathbf{u})=t_{k-1}(\mathbf{v})$ and $\Gamma_{k}(\mathbf{u})=\Gamma_{k}(\mathbf{v})$.

Therefore, upon defining a binary relation $\rho_{n}$ on $X_{n}^{+}$by $(\mathbf{u}, \mathbf{v}) \in \rho_{n}$ if and only if $\mathbf{u} \approx \mathbf{v}$ is an identity holding in $\mathcal{T}_{k}$, by well-known general-algebraic results [4] we obtain that $\rho_{n}$ is a (fully invariant) congruence of the free semigroup $X_{n}^{+}$, while $X_{n}^{+} / \rho_{n}$ is the free object of $\mathcal{T}_{k}$, freely generated by $X_{n} / \rho_{n}$ (where $\left|X_{n} / \rho_{n}\right|=n$ ). So, to each $\rho_{n}$-class containing a word $\mathbf{w}$ such that $|\mathbf{w}| \geqslant k$ it is possible to adjoin, in an injective fashion, a triple $(\Gamma, \mathbf{a}, \mathbf{b})$ consisting of a digraph $\Gamma$ (induced by any word from $\mathbf{w} / \rho_{n}$ ) and two of its vertices a, b (representing $h_{k-1}(\mathbf{w})$ and $t_{k-1}(\mathbf{w})$, respectively). Of course - as we shall shortly see - not every such triple is admissible.

In fact, given $k$ and $n$, there is a fixed digraph $B(n, k-1)$ of which any $\Gamma$ constructed as above is a subgraph. The vertex set of $B(n, k-1)$ is $X_{n}^{k-1}$, the set of all words of length $k-1$ over $X_{n}$, while edges are defined exactly as already described: $\mathbf{a} \rightarrow \mathbf{b}$ is an edge if and only if $\mathbf{a}=h_{k-1}(\mathbf{u})$ and $\mathbf{b}=t_{k-1}(\mathbf{u})$ for some word $\mathbf{u} \in X_{n}^{k}$. In the latter case, it is said that the edge $\mathbf{a} \rightarrow \mathbf{b}$ represents $\mathbf{u}$. 
A (directed) walk on a digraph $(V, E)$ is pseudo-Eulerian, if it contains each directed edge from $E$ at least once. (A closed pseudo-Eulerian walk is sometimes called a Chinese postman walk; see [1] for basic notions and background in digraph theory.) A directed graph that contains a pseudo-Eulerian walk must have at most one non-trivial (weakly) connected component, and the walk in question visits each vertex from that component at least once.

LemmA 2.2. (1) For any word $\mathbf{w} \in X_{n}^{+}$such that $|\mathbf{w}| \geqslant k$, the digraph $\Gamma_{k}(\mathbf{w})$ is a (weakly) connected subgraph of $B(n, k-1)$ that contains a pseudo-Eulerian walk.

(2) For any connected subgraph $H$ of $B(n, k-1)$ containing a pseudo-Eulerian walk, there exists a word $\mathbf{w}$ such that $|\mathbf{w}| \geqslant k$ and $\Gamma_{k}(\mathbf{w})=H$.

Proof. (1) is obvious from the definition of the graph $\Gamma_{k}(\mathbf{w})$.

(2) Let $\mathbf{a}_{0} \rightarrow \cdots \rightarrow \mathbf{a}_{p}$ be a pseudo-Eulerian walk in $H$. Then, for any $i>0$, we have $\mathbf{a}_{i}=t_{k-1}\left(\mathbf{a}_{i-1}\right) x_{m_{i}}$ for some $x_{m_{i}} \in X_{n}$. It is now straightforward to verify that

$$
\mathbf{w}=\mathbf{a}_{0} x_{m_{1}} \cdots x_{m_{p}}=\mathbf{a}_{0} t_{1}\left(\mathbf{a}_{1}\right) \cdots t_{1}\left(\mathbf{a}_{p}\right)=h_{1}\left(\mathbf{a}_{0}\right) \cdots h_{1}\left(\mathbf{a}_{p-1}\right) \mathbf{a}_{p}
$$

is a word with the required properties.

Hence, the elements of $\mathfrak{F}_{n}\left(\mathcal{T}_{k}\right)$ represented by words of length $\geqslant k$ can be identified with triples $(\Gamma, \mathbf{a}, \mathbf{b})$ such that $\mathbf{a}, \mathbf{b} \in V(\Gamma)$ and $\Gamma$ is a connected subgraph of $B(n, k-1)$ containing a pseudo-Eulerian walk from $\mathbf{a}$ to $\mathbf{b}$.

Note that the digraphs of the type $B(n, \ell)$ are well-known and widely investigated in graph theory and combinatorics in general: these are the so-called de Bruijn digraphs [7-9]. For example, $B(n, 2)$ is the complete digraph with loops on $n$ vertices, while for any $\ell \geqslant 2$, we have that $B(n, \ell+1)$ is the line graph of $B(n, \ell)$. Ultimately, we are going to utilise a result from the spectral theory of de Bruijn graphs in order to prove our main result.

\section{Estimating the free spectrum of $\mathcal{T}_{k}$.}

LEMMA 3.1.

$$
f_{n}\left(\mathcal{T}_{k}\right) \leqslant n^{2(k-1)} 2^{n^{k}}+\frac{n^{k}-n}{n-1}
$$

Proof. The second summand is equal to the sum $\sum_{m=1}^{k-1} n^{m}$ counting the number of words over $X_{n}$ of length $\leqslant k-1$. As we have seen in the previous section, if $|\mathbf{w}| \geqslant k$, then the classes $\mathbf{w} / \rho_{n}$ are in a one-to-one correspondence with triples $(\Gamma, \mathbf{a}, \mathbf{b})$, where $\Gamma$ is a connected subgraph of the de Bruijn digraph $B(n, k-1)$ possessing a pseudoEulerian walk from a to b. There are precisely $\left(n^{k-1}\right)^{2}$ ways to choose the pair $(\mathbf{a}, \mathbf{b})$, while a connected subgraph of a digraph $D$ is completely determined by a choice of edges from $E(D)$. Since $B(n, k-1)$ has a total of $n^{k}$ edges, an upper bound $2^{n^{k}}$ holds for the number of ways in which the subgraph $\Gamma$ can be selected.

Clearly, the upper bound provided by the above lemma is asymptotically equivalent to $n^{2(k-1)} 2^{n^{k}}$. Therefore, in the following we are going to look for a lower bound $d_{n, k} \leqslant f_{n}\left(\mathcal{T}_{k}\right)$ such that $d_{n, k} \sim n^{2(k-1)} 2^{n^{k}}$, thus completing the proof of Theorem 1.1. To achieve this, we follow a strategy (that resembles the one from [13]) of focusing to 
words inducing digraphs with closed pseudo-Eulerian walks. Recall that a digraph is strongly connected if there is a directed path between any pair of its vertices.

LEMMA 3.2. A connected digraph D has a closed pseudo-Eulerian walk if and only if it is strongly connected.

Proof. $(\Rightarrow)$ Since a pseudo-Eulerian walk of $D$ must traverse each directed edge at least once, by connectedness it follows that it visits each vertex at least once. Therefore, for any two $u, v \in V(D)$, there is a walk $u \rightsquigarrow v$ along the considered closed pseudoEulerian walk, and so there is a shortest walk from $u$ to $v$. Such a walk must be cycle-free thus it is a path.

$(\Leftarrow)$ Let $e_{1}, \ldots, e_{m}$ be the enumeration of all edges of $D$, where $e_{i}=\left(u_{i}, v_{i}\right)$ for $1 \leqslant i \leqslant m$. Since $D$ is assumed to be strongly connected, there are paths leading from $v_{i}$ to $u_{i+1}$ for all $1 \leqslant i<m$, and from $v_{m}$ to $u_{1}$. Now, we have a pseudo-Eulerian walk on $D$ comprising all these paths composed with the enumerated edges.

The following auxiliary result parallels [13, Lemma 4.1].

LEMMA 3.3. Let $H$ be a connected subgraph of $B(n, k-1)$ with $q$ vertices. If $H$ has a closed pseudo-Eulerian walk, then any maximal set of $\mathcal{T}_{k}$-nonequivalent words $\mathbf{w}$ with $\Gamma_{k}(\mathbf{w})=H$ has precisely $q^{2}$ elements.

Proof. If $\mathcal{T}_{k}$ fails to satisfy $\mathbf{w} \approx \mathbf{w}^{\prime}$, while $\Gamma_{k}(\mathbf{w})=\Gamma_{k}\left(\mathbf{w}^{\prime}\right)=H$, then $\mathbf{w}, \mathbf{w}^{\prime}$ must have either different prefixes, or different suffixes of length $k-1$. Since $|V(H)|=q$ and the prefixes and suffixes in question are vertices of $H$, there are at most $q^{2}$ words that are $\mathcal{T}_{k}$-nonequivalent and induce $H$. On the other hand, since $H$ has a closed pseudo-Eulerian walk, for any $\mathbf{u}, \mathbf{v} \in V(H)$, it has a pseudo-Eulerian walk from $\mathbf{u}$ to $\mathbf{v}$ : namely, we can traverse all edges of $H$ at least once by starting from $\mathbf{u}$ and returning to the same vertex by the given closed pseudo-Eulerian walk of $H$, and then proceed along the same walk to $\mathbf{v}$. Clearly, there is a word $\mathbf{w} \in X_{n}^{+}$corresponding to the walk just described; hence, we have a set of $q^{2}$ words not equivalent in $\mathcal{T}_{k}$ such that all words in the set induce the same digraph $H$.

The basic idea is to use the above lemma in the special case when $q=n^{k-1}$ : we shall see that (labelled) strongly connected subgraphs of $B(n, k-1)$ (which necessarily have the same vertex set as $B(n, k-1)$ itself) are numerous enough to yield the desired lower bound. To be more precise, our key observation can be formulated as follows.

Proposition 3.4. Let $\Delta_{n, k}$ denote the number of all strongly connected subgraphs of the de Bruijn digraph $B(n, k-1), k \geqslant 2$. Then $\Delta_{n, k} \sim 2^{n^{k}}$. In other words, a random subgraph of $B(n, k-1)$ (where edges are selected with probability $p=1 / 2$ ) is strongly connected with high probability as $n \rightarrow \infty$.

A crucial ingredient for the proof of this proposition comes from spectral graph theory. Namely, in [8] Delorme and Tillich provide bounds for eigenvalues and a number of spectral indices of de Bruijn graphs. For a graph $G$, call a partition $(S, V(G) \backslash$ $S$ ) of its vertex set a cut. The edge boundary $\partial S$ of $G$ with respect to this cut is the set of all edges $(u, v)$ such that $u \in S$ and $v \notin S$ (there is a natural analogue of this notion for undirected graphs, too). The isoperimetric number of $G, i(G)$, is defined as the minimum of the quotient $|\partial S| /|S|$ with $S$ ranging over all non-void subsets of $V(G)$ such that $|S| \leqslant|V(G)| / 2$. 
LEMma 3.5 ([8]). If $\ell \geqslant 2$ then

$$
i(B(n, \ell)) \geqslant \frac{n}{4(\ell-1)} .
$$

Actually, the lower bound that appears in Theorem 9 of [8] is twice the above one, $n /(2(\ell-1))$, but it concerns the 'undirected version' of $B(n, \ell)$ in which multiple edges and loops are allowed. Since $B(n, \ell)$ is an Eulerian digraph (i.e. the in-degree equals the out-degree at any vertex), it readily follows that a same number of directed edges cross an arbitrary cut in both directions. Therefore, $|\partial S|$ (and so the isoperimetric number) in $B(n, \ell)$ is half of that in its undirected twin. Also, the condition $\ell \geqslant 2$ is not mentioned in the original formulation of the cited result, but a careful reading of [8] shows that it must be assumed in order to have all calculations valid (and, of course, for the above quotient to be meaningful in the first place).

Proof of Proposition 3.4. Let $N_{n, k}$ be the number of all subgraphs of $B(n, k-1)$ with the same vertex set that are not strongly connected; then $\Delta_{n, k}+N_{n, k}=2^{n^{k}}$. If $G$ is such a graph, then there exists a cut $\left(S, X_{n}^{k-1} \backslash S\right)$ crossed by no edge of $G$. Since $B(n, k-1)$ is Eulerian, there is no loss of generality to assume that $|S| \leqslant n^{k-1} / 2$. For a fixed cut with the latter property, there are precisely $2^{n^{k}-|\partial S|}$ subgraphs of $B(n, k-1)$ with the same vertex set none of whose edges cross the considered cut (here, $\partial S$ denotes the edge boundary of $S$ in $B(n, k-1))$. By Lemma 3.5 we have

$$
|\partial S| \geqslant i(B(n, k-1))|S| \geqslant \frac{n|S|}{4(k-2)},
$$

provided $k \geqslant 3$. Note that this can be freely assumed, since the case $k=2$ has been already dealt with in [13]; alternatively, for $k=2$, we can use the obvious fact that $|\partial S|=|S|(n-|S|) \geqslant n|S| / 2$. In any case, for any $k \geqslant 2$, there exists a positive number $\beta$ (depending only on $k$ ) such that $|\partial S| \geqslant \beta n|S|$ holds for all the considered cuts.

Now, if $\left(S, X_{n}^{k-1} \backslash S\right)$ is an arbitrary cut in $B(n, k-1)$, write for brevity $s=|S|$ and $s^{*}=\min \left\{s, n^{k-1}-s\right\}$. We obtain the following estimate:

$$
\begin{aligned}
N_{n, k} & \leqslant \sum_{s=1}^{n^{k-1}-1}\left(\begin{array}{c}
n^{k-1} \\
s
\end{array}\right) 2^{n^{k}-\beta n s^{*}}=2^{n^{k}} \sum_{s=1}^{n^{k-1}-1}\left(\begin{array}{c}
n^{k-1} \\
s
\end{array}\right)\left(2^{-\beta n}\right)^{s^{*}} \\
& =2^{n^{k}} \sum_{s=1}^{n^{k-1}-1}\left(\begin{array}{c}
n^{k-1} \\
s^{*}
\end{array}\right)\left(2^{-\beta n}\right)^{s^{*}} \leqslant 2^{n^{k}+1} \sum_{s=1}^{\left.\frac{n^{k-1}+1}{2}\right\rfloor}\left(\begin{array}{c}
n^{k-1} \\
s
\end{array}\right)\left(2^{-\beta n}\right)^{s} .
\end{aligned}
$$

We use the well-known inequality $\left(\begin{array}{c}m \\ r\end{array}\right) \leqslant\left(\frac{m e}{r}\right)^{r}$ to establish that

$$
\left(\begin{array}{c}
n^{k-1} \\
s
\end{array}\right) \leqslant\left(\frac{n^{k-1} e}{s}\right)^{s} \leqslant\left(n^{k-1} e\right)^{s}=2^{\left((k-1) \log _{2} n+\log _{2} e\right) s} \leqslant\left(2^{\alpha \log _{2} n}\right)^{s}
$$

for a suitably chosen constant $\alpha$ (that depends only on $k$ ) and $n \geqslant 2$. Therefore, we have

$$
\frac{N_{n, k}}{2^{n^{k}}} \leqslant 2 \cdot \sum_{s=1}^{\left\lfloor\frac{n^{k-1}+1}{2}\right\rfloor}\left(2^{\alpha \log _{2} n-\beta n}\right)^{s}
$$


For $n$ large enough we are going to have $\alpha \log _{2} n-\beta n<0$, implying $\left(2^{\alpha \log _{2} n-\beta n}\right)^{s} \leqslant$ $2^{\alpha \log _{2} n-\beta n}$ for all $s \geqslant 1$ and thus

$$
\frac{N_{n, k}}{2^{n^{k}}} \leqslant\left(n^{k-1}+1\right) 2^{\alpha \log _{2} n-\beta n}=\frac{n^{\alpha}\left(n^{k-1}+1\right)}{2^{\beta n}} .
$$

It immediately follows that

$$
\lim _{n \rightarrow \infty} \frac{N_{n, k}}{2^{n^{k}}}=0
$$

that is, $\Delta_{n, k} \sim 2^{n^{k}}$.

Proof of Theorem 1.1. An upper bound for $f_{n}\left(\mathcal{T}_{k}\right)$ is provided by Lemma 3.1. As for the lower bound, Lemma 3.3 implies that for any strongly connected subgraph of $B(n, k-1)$ there are $n^{2(k-1)}$ words not equivalent in $\mathcal{T}_{k}$ that induce the subgraph in question. Hence, $f_{n}\left(\mathcal{T}_{k}\right) \geqslant n^{2(k-1)} \Delta_{n, k}$, where $\Delta_{n, k}$ is the number of strongly connected subgraphs of $B(n, k-1)$. Now Proposition 3.4 completes the proof of the theorem.

REMARK 3.6. In this note, we used the definition of $k$-testability that appears in [22], and that originated from [3]. This variant of $k$-testability is consistent with [15, 21] so that $\mathcal{A}$ is indeed the variety of 2 -testable semigroups. An alternative definition of $k$-testability is featured in [20] and several other references, but the impact of this alternative is minor to our main result: under the modified definition, the asymptotic class of the resulting free spectrum is $n^{2 k} 2^{n^{k}}$.

ACKNOWLEDGEMENTS. I am indebted to my colleagues Miloš Stojaković and Petar Markovic for valuable conversations concerning the present topic. The support of the Ministry of Science and Technological Development of the Republic of Serbia (through Grant No. 174019) is gratefully acknowledged.

\section{REFERENCES}

1. J. Bang-Jensen and G. Z. Gutin, Digraphs: Theory, algorithms and applications, 2nd edn. (Springer-Verlag, New York, 2010).

2. J. Berman, Free spectra gaps and tame congruence types, Int. J. Algebra Comput. 5 (1995), 651-672.

3. J. A. Brzozowski and I. Simon, Characterizations of locally testable events, Discrete Math. 4 (1973), 243-271.

4. S. Burris and H. P. Sankappanavar, A course in universal algebra (Springer-Verlag, New York, 1981).

5. S. Crvenković, I. Dolinka and N. Ruškuc, Finite semigroups with few term operations, J. Pure Appl. Algebra 157 (2001), 205-214.

6. S. Crvenković, I. Dolinka and N. Ruškuc, The Berman conjecture is true for finite surjective semigroups and their inflations, Semigroup Forum 62 (2001), 103-114. $758-764$

7. N. G. de Bruijn, A combinatorial problem, Nederl. Akad. Wetensch., Proc. 49 (1946),

8. C. Delorme and J.-P. Tillich, The spectrum of de Bruijn and Kautz graphs, Eur. J. Comb. 19 (1998), 307-319.

9. I. J. Good, Normal recurring decimals, J. Lond. Math. Soc. 21 (1946), 167-169.

10. S. Hermiller, D. F. Holt and S. Rees, Groups whose geodesics are locally testable, Int. J. Algebra Comput. 18 (2008), 911-923. 
11. G. Higman, The orders of relatively free groups, in Proceedings of International Conference on the Theory of Groups, Canberra, 1965 (Gordon \& Breach, New York, 1967), $153-165$.

12. D. Hobby and R. McKenzie, The structure of finite algebras, Contemporary Mathematics, vol. 76 (American Mathematical Society, Providence, RI, 1988).

13. K. Kátai-Urbán and Cs. Szabó, On the free spectrum of the variety generated by the combinatorial completely 0-simple semigroups, Glasgow Math. J. 49 (2007), 93-98.

14. S. Kim and R. McNaughton, Computing the order of a locally testable automaton, SIAM J. Comput. 23 (1994), 1193-1215.

15. E. W. H. Lee and M. V. Volkov, On the structure of the lattice of combinatorial ReesSushkevich varieties, in Semigroups and formal languages, Lisbon, 2005 (André J. M. et al., Editors) (World Scientific, Hackensack, NJ, 2007), 164-187.

16. D. B. McAlister and F. Soares, One-dimensional tiling semigroups and factorial languages, Comm. Algebra 37 (2009), 276-295. 1971).

17. R. McNaughton and S. Papert, Counter-free automata (M.I.T. Press, Cambridge, MA,

18. P. M. Neumann, Some indecomposable varieties of groups, Q. J. Math. (Oxford) 14(2) (1963), 46-50.

19. J.-E. Pin, Finite semigroups and recognizable languages: An introduction, in Semigroups, formal languages and groups (Fountain J., Editor) (NATO Advanced Study Institute \& Kluwer, Dordrecht, 1995), 1-32. 309-317.

20. A. N. Trahtman, The varieties of $n$-testable semigroups, Semigroup Forum 27 (1983),

21. A. N. Trahtman, Identities of a five-element 0-simple semigroup, Semigroup Forum $\mathbf{4 8}$ (1994), 385-387.

22. A. N. Trahtman, Identities of locally testable semigroups, Comm. Algebra 27 (1999), $5405-5412$. 